\title{
EMG Analysis Using Wavelet Functions To Determine Muscle Contraction
}

\author{
M. B. I. Reaz, M. S. Hussain \\ Dept. of Electrical and Computer Engineering \\ International Islamic University Malaysia \\ Gombak, Malaysia \\ mamun.reaz@iiu.edu.my
}

\author{
F. Mohd-Yasin \\ Faculty of Engineering \\ Multimedia University \\ Cyberjaya, Malaysia
}

\begin{abstract}
Modern spectrum analysis has been applied to various bio-medical signal processing. Wavelet transform (WT) has been applied in this research for the analysis of the surface electromyography signal (SEMG). Mean and median frequencies of the SEMG power spectrum were used to indicate changes in muscle contraction during gait. This paper reports on the effectiveness of the wavelet transform applied to the SEMG signal as a means of understanding muscle contractions during gait. Power spectrum analysis on the EMG signal from right rectus femoris muscle was performed using various wavelet functions (WF). With the appropriate choice of the WF, it is possible to analyze SEMG significantly. Results show that WF Daubechies45 presents the most significant changes in SEMG power spectrum compared to the other WFs.
\end{abstract}

Keywords- EMG, Motor unit, Power spectrum, Wavelet transform

\section{INTRODUCTION}

Electromyography (EMG) signal represents the electrical activity of muscles. A muscle is composed of many Motor Units (MUs). EMG signals detected directly from the muscle or from the skin by using surface electrodes show a train of motor unit action potentials (MUAP) plus noise. With increasing muscle force, the raw EMG signal shows an increase in the number of MUAP recruited at increasing firing rates. EMG signals are modeled as a zero mean colored noise, which can be characterized by power spectrum density function [1]. It is desired to apply a method of power spectrum analysis to study the frequency characteristics of random signals like EMG. In the SEMG, recruitment and increase in the firing frequencies are seen as an amplitude increase of the SEMG signal [2]. Changes in the EMG power spectrum are used as an indicator of changes in muscle contraction and muscle fatigue for ergonomic purposes [3].

In this research, SEMG are decomposed using Discrete Wavelet Transform (DWT) with various WFs. It is the technique that provides information related to the timefrequency variation of the signal and used for feature enhancement for biosignals. The purpose of this research is to analyze the SEMG power spectral parameters with the various WF during nine trial walk. In this study WFs, Haar, Daubechies (db2, db3, db4, db5, db45) and Symmlet (sym4, sym5) are used for the WT. As a tool for analyzing frequency components of the EMG signals, Fast Fourier Transform (FFT) is considered.

A superposed EMG signal can be considered as summation of sine waves with different frequency velocity. The FFT algorithm is described as a decomposition of the EMG signal to its underlined sinus contents. The mean and median frequencies are the most important parameters for analyzing the frequency components of the EMG signal.

In this research, mean and median frequency parameters are considered for the EMG power spectrum analysis during a 9 trial walk. Results show that, WF db45 presents the best contrast in the mean and median frequencies while compared to the other WFs. The study also demonstrates that mean and median frequency increases with an increase of muscle contraction.

\section{MTHODOLOGY}

In this experiment, 11 separate EMG data files were used. Raw EMG signals were collected from Motion Lab Systems, Inc. [4] for the simulation of the algorithm. SEMG was recorded from the right "rectus femoris" muscle of a normal subject (teenage male). All analog channels are recorded at 800 samples per second.

The 11 sample EMG data were the 9 trial walks, muscle at rest level and muscle at maximum contraction level. These SEMG signals were decomposed using DWT with eight different wavelet functions (haar, db2, db3, db4, db4, db45, sym4 and sym5). The DWT was implemented using MATLAB Wavelet toolbox.

The power spectrum properties (mean frequency and median frequency) were calculated to estimate the muscle contraction at various walking trials. Mean and median frequency of the muscle's resting stage and maximum effort stage were also calculated. Figure 1 shows the algorithm flow for the power spectrum analysis.

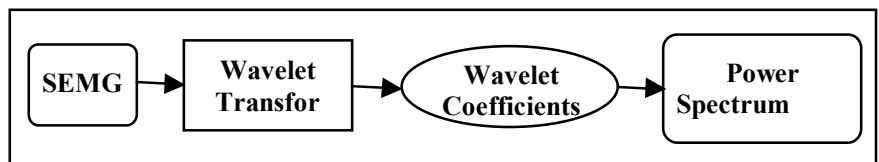

Fig. 1 Flow of the algorithm for the power spectrum analysis 


\section{A. Wavelet Decomposition}

The DWT is computed by successive low pass and high pass filtering of the discrete time domain signal. The DWT of a signal $x[n]$ is calculated by passing it through a series of filters. First the samples are passed through a low pass filter with impulse response $g[n]$ resulting in a convolution of the two as given in equation 1.

$$
y[n]=x[n]^{*} g[n]=\sum_{k=-\infty}^{\infty} x[k] \cdot g[n-k]
$$

The signal is also decomposed simultaneously using a highpass filter $h[n]$. The outputs giving the detail coefficients (from the high-pass filter) and approximation coefficients (from the low-pass). The filter outputs are then down-sampled (or subsampled) as given in equation 2 and 3 :

$y_{\text {low }}[n]=\sum_{k=-\infty}^{\infty} x[k] \cdot g[2 n-k]$

$$
y_{\text {high }}[n]=\sum_{k=-\infty}^{\infty} x[k] \cdot h[2 n-k]
$$

The ability of DWT to extract features from the signal is dependant on the appropriate choice of the mother wavelet function. Even though there is no well-defined rule for selecting a wavelet basis function in a particular application or analysis, some properties of the wavelets make a specific mother wavelet more suitable for a given application and signal type $[1,5]$.

\section{B. Power Spectrum Analysis}

The power spectrum was obtained using FFT techniques. Hanning window was used with a 128 point FFT. The discrete fourier transform of a discrete signal with $\mathrm{N}$ samples is given in equation 4 .

$$
f(k)=\sum_{n=0}^{N-1} f(n) e^{\frac{-i 2 \pi k}{N} n} ; \text { for } 0 \leq k<N
$$

The mean frequency was obtained from the power spectrum and given in equation $5[6]$.

$$
p f_{\text {mean }}=\frac{\int f P S(f) d f}{\int P S(f) d f}
$$

The median frequency was calculated by finding the middle frequency value of the power spectrum.

\section{RESULTS AND DISCUSSION}

The difference of mean and median frequency is used to analyze the EMG signal to understand the muscle contractions. Table 1 represents the mean frequency and table 2 represents the median frequency of the WT for eight different WFs during the nine walking trials.

Figure 2 and figure 3 shows the graphical representation of the mean and median frequency obtained from table 1 and table 2. According to figure 2 and figure $3, \mathrm{WFs}$ haar, db2, db3, db4, $\mathrm{db} 5$, sym 4 and sym5 show similar results for both mean and median power frequency in $50 \mathrm{~Hz}$ range. The wavelet function $\mathrm{db} 45$ presents higher mean (1and median frequency values compared to the other seven WFs within 50 to $70 \mathrm{~Hz}$ range. Figure 2 and 3 depicts that $\mathrm{db} 45$ can represent more variations on the power spectrum while analyzing SEMG signals. Changes in the mean and median frequencies are most significant in $\mathrm{db} 45$ to indicate muscle contraction compared to the other seven WFs. The significant increase in mean and median frequency indicates the presence of newly recruited Motor Units (MUs) during the nine trial walking procedures.

TABLE I. MEAN POWER FREQUENCY

\begin{tabular}{lllllllll}
\hline \hline & Haar & $\mathrm{db2}$ & $\mathrm{db} 3$ & $\mathrm{db} 4$ & $\mathrm{db5}$ & $\mathrm{db45}$ & sym4 & sym5 \\
\hline Trial 1 & 51.88 & 50.66 & 53.02 & 50.22 & 51.1 & 65.17 & 51.25 & 50.59 \\
Trial 2 & 52.86 & 51.77 & 54.48 & 51.73 & 51.88 & 63.16 & 49.47 & 52.98 \\
Trial 3 & 48.32 & 48.32 & 47.12 & 52.22 & 52.06 & 53.42 & 47.28 & 48.23 \\
Trial 4 & 48.64 & 50.19 & 49.785 & 49.365 & 48.855 & 61.006 & 52.028 & 49.332 \\
Trial 5 & 52.257 & 51.68 & 49.33 & 47.942 & 48.742 & 56.797 & 52.785 & $52(037)$ \\
Trial 6 & 50.259 & 46.861 & 50.577 & 49.468 & 52.963 & 60.405 & 50.215 & 49.59 \\
Trial 7 & 50.6 & 52.618 & 53.595 & 49.144 & 54.537 & 64.798 & 49.281 & 50.151 \\
Trial 8 & 50.302 & 50.302 & 47.335 & 50.132 & 51.384 & 66.019 & 49.07 & 47.959 \\
Trial 9 & 48.247 & 49.146 & 50.743 & 48.891 & 51.411 & 64.553 & 48.194 & 47.771 \\
\hline \hline
\end{tabular}

TABLE II. MEDIAN POWER FREQUENCY

\begin{tabular}{lllllllll}
\hline \hline & \multicolumn{1}{c}{ Haar } & \multicolumn{1}{c}{$\mathbf{d b 2}$} & $\mathrm{db} 3$ & \multicolumn{1}{c}{$\mathrm{db}$ 4 } & $\mathrm{db5}$ & $\mathrm{db45}$ & $\mathrm{sym4}$ & sym5 \\
\hline Trial 1 & 53.13 & 51.56 & 54.69 & 50.0 & 51.56 & 70.31 & 51.56 & 50.0 \\
Trial 2 & 54.69 & 53.13 & 57.81 & 53.13 & 54.69 & 68.75 & 48.44 & 54.69 \\
Trial 3 & 46.88 & 46.88 & 45.31 & 54.69 & 54.69 & 57.81 & 45.31 & 46.88 \\
Trial 4 & 48.44 & 50.0 & 50.0 & 51.563 & 48.438 & 65.625 & 53.125 & 48.438 \\
Trial 5 & 54.688 & 53.125 & 50.0 & 46.875 & 50.0 & 59.375 & 54.688 & 53.125 \\
Trial 6 & 51.563 & 46.875 & 51.563 & 51.563 & 56.25 & 65.625 & 51.553 & 50.0 \\
Trial 7 & 51.563 & 54.688 & 56.25 & 49.144 & 59.375 & 68.75 & 50.0 & 51.563 \\
Trial 8 & 50.0 & 50.0 & 48.438 & 50.0 & 53.125 & 71.875 & 50.0 & 46.875 \\
Trial 9 & 45.313 & 48.438 & 53.125 & 48.438 & 53.125 & 70.313 & 48.438 & 48.438 \\
\hline \hline
\end{tabular}

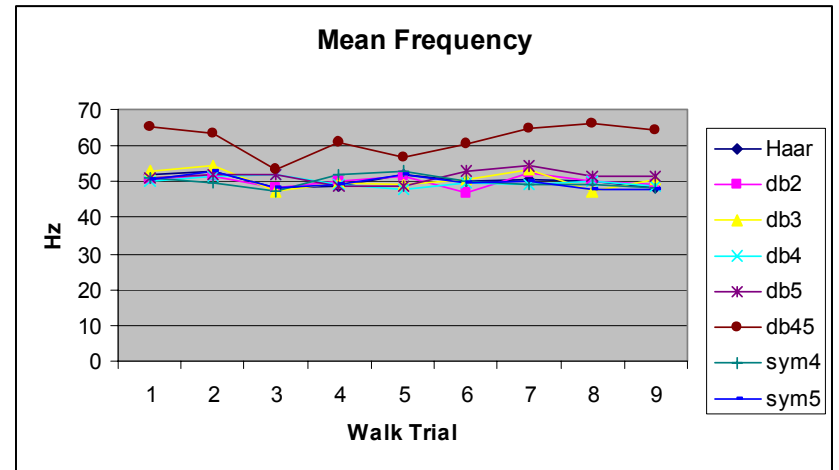

Fig. 2 Mean power frequency at different wavelet functions 
According to the study by Hagberg and Ericson, mean power frequency was lower at low contraction levels when compared with high contraction levels [7]. Moritani et al. also obtained similar results where significant increase in SEMG amplitude and mean power frequency were found with increasing force [8]. It is also shown that during muscle fatigue, the power spectrum of SEMG shows a shift to lower frequencies [9]. Median frequency is used to quantify this shift. The cause of this is related to the changes in the MU and changes in the firing characteristics of the MU [10].

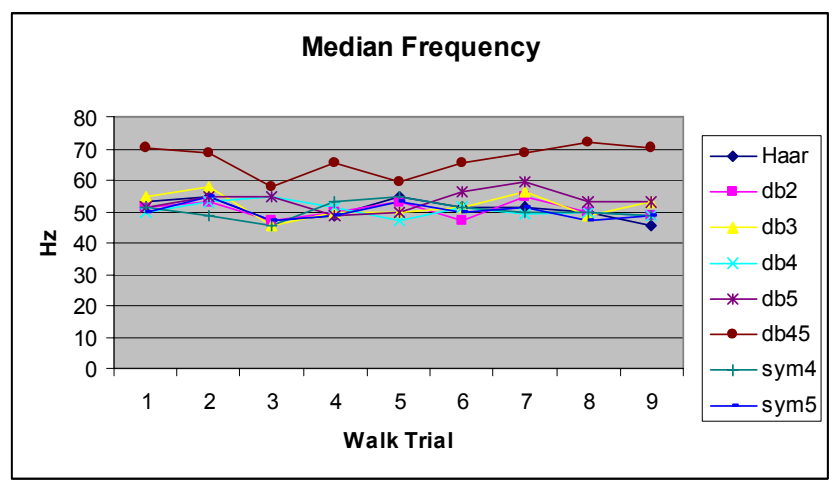

Fig. 3 Median power frequency at different wavelet functions

Results obtained by this research indicate that there was significant increase in the mean and median frequency from the resting state of the muscle to maximum contraction level by using wavelet $\mathrm{db} 45$ as in [7]. This increasing change of mean and median frequency is illustrated in figure 4 and figure 5 . Based on results, figure 4 and figure 5 highlight that the mean and median frequency in figure 2 and figure 3 decreased from trial 1 to trial 3 indicating that there was a decrease of Motor Units (MUs) recruitment as in [10]. The frequency increased from trial 3 to trial 4 and dropped again in trial 5. After trial 5 both the mean and median frequency increased representing significant change in the MU's as in $[8,10]$.

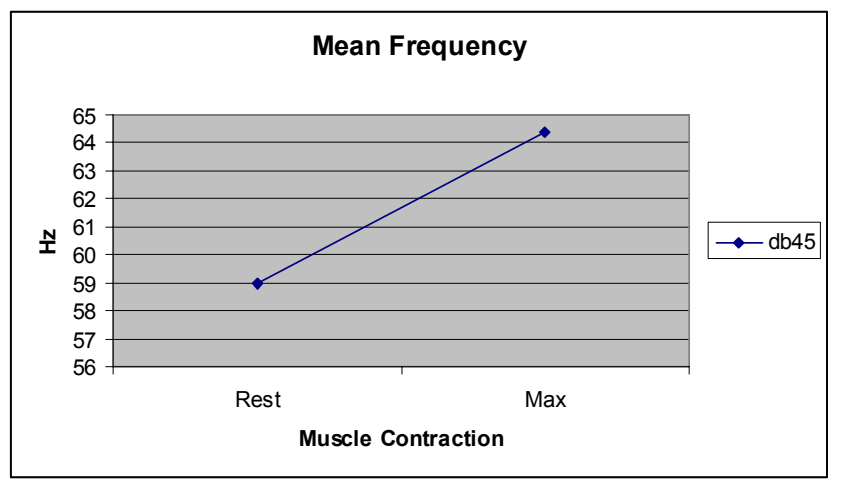

Fig. 4 Mean power frequency at rest and maximum contraction stage using $\mathrm{db} 45$ wavelet function

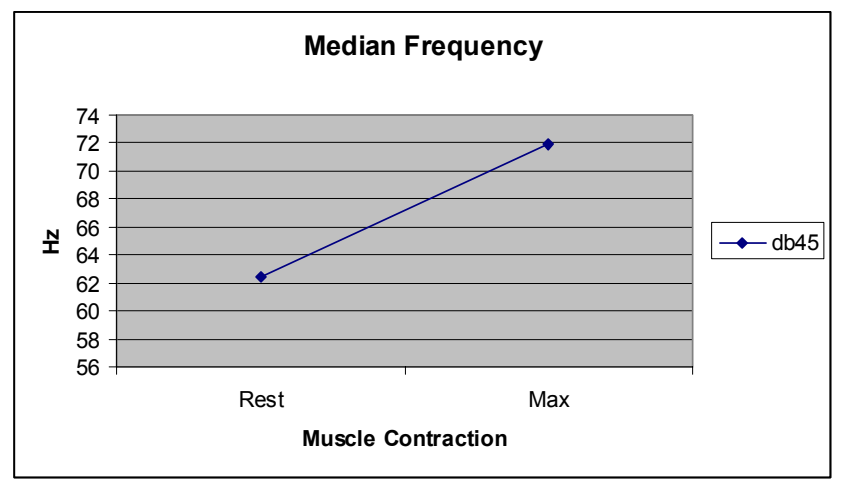

Fig. 5 Median power frequency at rest and maximum contraction stage using $\mathrm{db} 45$ wavelet function

\section{CONCLUSION}

This article demonstrates that WF db45 most significantly presents the changes in the power spectrum properties (mean and median frequency) representing the differences in muscle contraction during gait. The study also shows that increase in the muscle contraction level (rest to max) leads to and increase in mean and median frequencies demonstrating changes in the MUs recruitment.

\section{REFERENCES}

[1] Reaz M. B. I., Hussain, M. S., Yasin M. F., "Techniques of EMG Signal Analysis: Detection, Processing, Classification and Applications", Biological Procedures Online, 2006, (accepted).

[2] Milner Brown H. S., Stein R. B.; "The relationship between the surface electromyogram and muscular force"; J of Physiol (Lond); vol. 246; pp 549-569; 1975

[3] Lindstrom L.,Kadefors R. Petersen I.; "An electromyographic index for localized muscle fatigue"; J of Appl Physiol: Respirat Environ Exercise Physol; vol. 43; pp 750-754; 1977

[4] Motion Lab website; http://www.emgsrus.com [last visited 09 Dec 05]

[5] Kumar D. K., Pah N. D., Bradley A.; Wavelet analysis of surface electromyography to determine muscle fatigue; IEEE Trans on Neural Syst Rehabil Eng.; vol. 11; No. 4; pp 400-406; Dec 2003

[6] Winter D. A.; Rau G.; Kadefors R.; Units, terms and standards in reporting of electromyographical research. Proceedings of the 4th Congress of ISEK; pp 100-101; Aug 5-10, 1979; Boston, USA

[7] Hagberg M. and Ericson B. E.; "Myoelectric power spectrum dependence of muscular contraction level of elbow flexors"; Eur J of Appl Physiol; vol. 48; issue 2, pp 147-156; 1982

[8] Moritani T., Nagata A., Muro M.; "Electromyographic manifestations of muscular fatigue"; Med Sci Sport Exerc; vol. 14; pp 198-202; 1982

[9] De Luca C. J.; "Myoelectrical manifestations of localized muscular fatigue in humans; CRC critical reviews in Biomedical Engineering; vol. 11; issue 4; pp 251-279; 1985

[10] Hermens H. J., Bruggen T. V.; "Simulation of fatigue related changes in spectral parameters of surface EMG"; IEEE $10^{\text {th }}$ Annual International Conference on Medicine and Biology Society; vol.4; pp. 1706-1707; U.S.A., Nov 4-7, 1988 